\title{
Significant inverse magnetocaloric effect induced by quantum criticality
}

\author{
Tao Liu,,${ }^{1,2,{ }^{*}}$ Xin-Yang Liu, ${ }^{2,}{ }^{*}$ Yuan Gao, ${ }^{2}$ Hai Jin,,${ }^{3}$ Jun He, ${ }^{1}$ Xian-Lei Sheng $\odot,{ }^{2}$ \\ Wentao Jin, ${ }^{2}$ Ziyu Chen $\odot,{ }^{2, \dagger}$ and Wei $\mathrm{Li}^{2,4,5, \$}$ \\ ${ }^{1}$ School of Science, Hunan University of Technology, Zhuzhou 412007, China \\ ${ }^{2}$ School of Physics, Beihang University, Beijing 100191, China \\ ${ }^{3}$ Department of Astronomy, Tsinghua Center for Astrophysics, Tsinghua University, Beijing 100084, China \\ ${ }^{4}$ International Research Institute of Multidisciplinary Science, Beihang University, Beijing 100191, China \\ ${ }^{5}$ Institute of Theoretical Physics, Chinese Academy of Sciences, Beijing 100190, China
}

(Received 9 March 2021; revised 1 June 2021; accepted 7 July 2021; published 27 July 2021)

\begin{abstract}
The criticality-enhanced magnetocaloric effect (MCE) near a field-induced quantum critical point (QCP) in the spin systems constitutes a very promising and highly tunable alternative to conventional adiabatic demagnetization refrigeration. Strong fluctuations in the low- $T$ quantum critical regime can give rise to a large thermal entropy change and thus significant cooling effect when approaching the QCP. In this work, through efficient and accurate many-body calculations, we show there exists a significant inverse MCE (iMCE) in the spin-1 quantum chain materials $\left(\mathrm{CH}_{3}\right)_{4} \mathrm{NNi}\left(\mathrm{NO}_{2}\right)_{3}(\mathrm{TMNIN})$ and $\mathrm{NiCl}_{2}-4 \mathrm{SC}\left(\mathrm{NH}_{2}\right)_{2}(\mathrm{DTN})$, where DTN has substantial low- $T$ refrigeration capacity while requiring only moderate magnetic fields. The iMCE characteristics, including the adiabatic temperature change $\Delta T_{\text {ad }}$, isothermal entropy change $\Delta S$, differential Grüneisen parameter, and the entropy change rate, are obtained with quantum many-body calculations at finite temperature. The cooling performance, i.e., the efficiency factor and hold time, of the two compounds is also discussed. Based on the many-body calculations on realistic models for the spin-chain materials, we conclude that the compound DTN constitutes a very promising and highly efficient quantum magnetic coolant with pronounced iMCE properties. We advocate that such quantum magnets can be used in cryofree refrigeration for space applications and quantum computing environments.
\end{abstract}

DOI: 10.1103/PhysRevResearch.3.033094

\section{INTRODUCTION}

The magnetocaloric effect (MCE) represents a significant adiabatic temperature change of a magnet as a response to the varying external magnetic fields [1-4]. Historically, the first sub-Kelvin regime cooling was realized through adiabatic demagnetization refrigeration (ADR) [5]. Recently, low- $T$ magnetic refrigeration has received refreshed research interest due to its important applications in space technology $[6,7]$ and the cryofree sub-Kelvin environment for quantum computers [8]. It is of great research interest to pursue novel MCE refrigerants that provide higher cooling powers and can reach lower temperatures. Among others, the quantum spin-chain materials with enhanced MCE characterized by the universally diverging Grüneisen ratio near the quantum critical point (QCP) [9-15] has been proposed as a very promising quantum critical coolant for magnetic refrigeration $[16,17]$ and an excellent alternative to the conventional ADR.

\footnotetext{
${ }^{*}$ These authors contributed equally to this work.

$\dagger$ chenzy@buaa.edu.cn

${ }^{\ddagger}$ w.li@buaa.edu.cn
}

Published by the American Physical Society under the terms of the Creative Commons Attribution 4.0 International license. Further distribution of this work must maintain attribution to the author(s) and the published article's title, journal citation, and DOI.
In most magnetic materials, the spin degrees of freedom in the system eventually "solidify" into a long-range order as it is cooled down to sufficiently low temperatures. In such magnetically ordered phase, the spin states are practically nontunable by external fields (due to the existence of giant Weiss molecular fields) and the corresponding MCE, temperature, or entropy change as a response to fields are usually negligible. Paramagnetic salts, such as $\mathrm{CrK}\left(\mathrm{SO}_{4}\right)_{2} \cdot 12 \mathrm{H}_{2} \mathrm{O}$ (chromic potassium alum, $\mathrm{CPA}$ ) and $\mathrm{Fe}\left(\mathrm{SO}_{4}\right)_{2} \mathrm{NH}_{4} \cdot 2 \mathrm{H}_{2} \mathrm{O}$ (ferric ammonium alum, FAA), etc., that host nearly noninteracting spins and do not order at very low temperatures, are widely used in ADR as a spin "gas" refrigerant [7]. It is commonly believed that the spin interactions are "harmful" for good ADR coolants, as they usually lead to magnetic ordering as $T$ lowers and spoil the MCE properties.

Nevertheless, there is an exotic exception to this classic fate of interacting quantum spins. In low-dimensional quantum magnets [18], the quantum fluctuations can be strong enough to prevent the spins from classical ordering even at $T=0$. One prominent case is the field-induced QCP in the correlated quantum magnetic materials. The enhanced quantum fluctuations near the QCP can significantly influence the thermodynamics in the quantum critical regime at low temperature [19]. When approaching the QCP, the spin system would experience a significant isothermal entropy change, which can be translated into a considerable temperature decrease under the adiabatic condition. Such quantum criticality-enhanced 
MCE is reflected in a diverging Grüneisen parameter [adiabatic temperature change rate; cf. Eq. (2)], $\Gamma_{B} \sim T^{-1 / z v}$, under external field $B$, with $z$ and $v$ the dynamical and critical exponents related to the universality class of the QCP [11,12]. Notably, such intriguing quantum critical phenomena in lowtemperature thermodynamics also provide a sensitive probe of QCP in experiments [20].

The low-temperature thermodynamic properties of a typical one-dimensional (1D) quantum magnetic systemthe spin-1/2 Heisenberg chain (HAFC) - have been intensively explored, where a pronounced $\mathrm{MCE}$ was predicted $[9,10]$ and also observed in the compound $\left[\mathrm{Cu}\left(\mu-\mathrm{C}_{2} \mathrm{O}_{4}\right)(4-\right.$ aminopyridine $\left.)_{2}\left(\mathrm{H}_{2} \mathrm{O}\right)\right]_{n}(\mathrm{CuP})$. The lowest achievable temperature with the spin-chain coolants has no principal limitation (as long as the interchain interactions are negligible), and the high efficiency factor as well as long hold time make the spin-1/2 HAFC materials very promising quantum critical refrigerants $[16,17]$.

Nevertheless, there is still plenty of room for further improvement of the performance of quantum magnetic refrigerants. As reported in Ref. [16], for the spin-1/2 HAFC compound $\mathrm{CuP}$, one has to start from a rather high magnetic field, e.g., $7 \mathrm{~T}$, significantly above the critical field $B_{c} \simeq 4.09 \mathrm{~T}$. In $\mathrm{CuP}$, there indeed exists significant MCE in the range $B>B_{c}$, while in the smaller-field side, i.e., $B \in\left[0, B_{c}\right]$, rather weak (inverse) MCE was observed. Therefore, the generated strong magnetic fields (of $7 \mathrm{~T}$ ) have actually not been fully exploited in the case of the spin-1/2 compound $\mathrm{CuP}$. On the other hand, popular paramagnetic refrigerants are typically with high spin $S \geqslant 1$, as larger entropy changes are generally expected for higher spin systems. For example, CPA and FAA are with $S=3 / 2$ and 5/2, respectively, and the gadolinium gallium garnet (GGG) is even with $S=7 / 2$. However, there are only a few studies of quantum spin models [13] and materials [15] with spin higher than $S=1 / 2$.

In this work, we systematically investigate the inverse MCE (iMCE) properties of the $S=1$ quantum chain models and materials with many-body simulations. Special emphases are put on two typical spin-1 HAFC materials, i.e., $\left(\mathrm{CH}_{3}\right)_{4} \mathrm{NNi}\left(\mathrm{NO}_{2}\right)_{3}(\mathrm{TMNIN})$ and $\mathrm{NiCl}_{2}-4 \mathrm{SC}\left(\mathrm{NH}_{2}\right)_{2}(\mathrm{DTN})$, whose magnetothermodynamic properties can be accurately simulated by the thermal-state linearized tensor renormalization group (LTRG) approaches [21,22]. In particular, we find that the compound DTN has iMCE refrigeration capacity comparable to the MCE of the spin-1/2 HAFC material $\mathrm{CuP}$, while requiring only a moderate external magnetic field of $B_{c} \simeq 3 \mathrm{~T}$ in the cooling process, which is much smaller than the latter requires. Due to the pronounced cooling effects and excellent thermal transport properties [23] in DTN, we propose that DTN constitutes a very promising iMCE refrigerant with only a moderate magnetic field and competitive performance, very suitable for practical refrigeration applications.

The remaining parts of the article are arranged as follows. We present the spin-1 chain models, their thermal manybody simulations, and the related spin-1 materials in Sec. II. Our main results on iMCE of the spin-1 chains are shown in Sec. III, and Sec. IV is devoted to the conclusion and outlook.

\section{SPIN-1 HEISENBERG-CHAIN MODEL AND MATERIALS}

\section{A. Heisenberg antiferromagnetic chain and tensor renormalization group}

The $S=1$ HAFC systems with single-ion anisotropy can be described by the Hamiltonian

$$
\mathcal{H}=\sum_{i}\left[J \vec{S}_{i} \cdot \vec{S}_{i+1}+D\left(S_{i}^{z}\right)^{2}+g \mu_{\mathrm{B}} B S_{i}^{z}\right],
$$

where $\vec{S}_{i}$ is the spin-1 operator on site $i$ (with $S_{i}^{z}$ its $z$ component), $J$ is the nearest-neighbor Heisenberg interaction, and $D$ is the single-ion anisotropy parameter. In the Zeeman term, $B$ is the external magnetic field, $g$ is the electronic Landé factor, and $\mu_{B}$ is the Bohr magneton.

In a rather wide parameter regime [24,25], the spin-1 chain model given by Eq. (1) has a nonmagnetic ground state with finite spin gap, which can be closed by applying a magnetic field $B$ through the field-induced quantum phase transition. In particular, for the case $D=0$, the model in Eq. (1) reduces to the isotropic spin-1 HAFC model with the renowned Haldane gap, $\Delta \approx 0.41 J$ [26-28], where the first-excited state is in a spin triplet $S=1$ sector. The introduction of $D$ in the spin chain can alter the size of the Haldane gap and drive the system into a trivial large- $D$ phase through a topological quantum phase transition at $D / J=0.93$ [29]. For $D>D_{c}$, the gap reopens and scales proportionally with $D$ in the large- $D$ limit. In addition, other off-diagonal single-ion anisotropies are found to be small in the two spin-1 chain compounds-TMNIN and DTN-considered in the present work, and we thus set them to zero in the rest of our discussion.

To simulate the finite-temperature properties and characterize the iMCE properties of the spin-1 chain materials, we employ the infinite-size LTRG method [21] in the bilayer form [22] for high-precision thermal many-body calculations down to low temperature $T / J=0.01$. In the process of imaginarytime evolution (cooling), we retain up to $\chi=400$ bond states (with truncation error $\epsilon \lesssim 10^{-5}$ ) in the matrix-product thermal density operator, which always guarantees accurate and converged thermodynamic results.

\section{B. iMCE property and performance characteristics}

Driven by external magnetic fields, the spin-1 chains in the quantum critical regime show significantly enhanced MCE. To quantitatively characterize the MCE property, we calculate the magnetic Grüneisen parameter,

$$
\Gamma_{B}=\frac{1}{T}\left(\frac{\partial T}{\partial B}\right)_{S}=-\frac{1}{C_{B}}\left(\frac{\partial M}{\partial T}\right)_{B},
$$

with $C_{B}=T(\partial S / \partial T)_{B}$ the magnetic specific heat (under an external field $B$ ). The Grüneisen parameter $\Gamma_{B}$ measures the temperature change rate as a response to the small variation of the external field under an adiabatic condition. In the numerator of Eq. (2) is a related differential quantity,

$$
\Theta_{T}=\left(\frac{\partial S}{\partial B}\right)_{T}=\left(\frac{\partial M}{\partial T}\right)_{B},
$$

which measures the isothermal entropy change rate. 


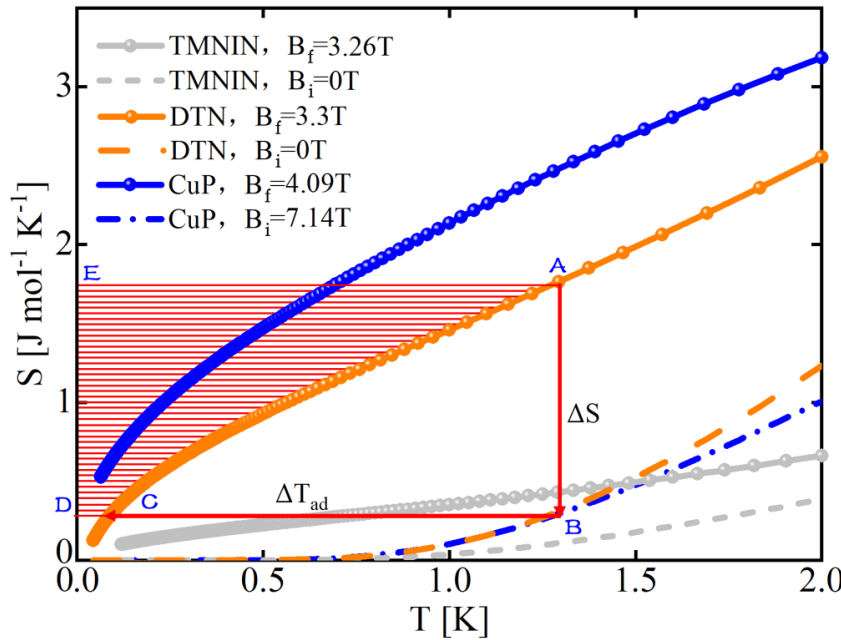

FIG. 1. Simulated thermal entropy $S(T)$ curves of three spinchain materials, under different initial fields $B_{i}$ and final fields $B_{f}=$ $B_{c}$. Entropy change $\Delta S$ (indicated by the vertical red arrow) and adiabatic temperature change $\Delta T_{\text {ad }}$ (horizontal red arrow) are shown explicitly for the compound DTN. A-B-C-A constitutes a single-shot refrigeration process, and the dashed regime $\mathrm{ACDE}$ represents the heat absorbed from the load in the isofield CA process.

Correspondingly, when integrated over a given range of fields, e.g., $B \in\left[B_{i}, B_{f}\right]$ with $B_{i}$ and $B_{f}$ the initial and final (critical) fields in the iMCE process, respectively, the isothermal entropy change is

$$
\Delta S(T)=\int_{B_{i}}^{B_{f}}\left(\frac{\partial S}{\partial B}\right)_{T} d B=\int_{B_{i}}^{B_{f}}\left(\frac{\partial M}{\partial T}\right)_{B} d B,
$$

and the adiabatic temperature change is

$$
\Delta T_{\mathrm{ad}}=\int_{B_{i}}^{B_{f}} \Gamma_{B} T d B=-\int_{B_{i}}^{B_{f}} \frac{T}{C_{B}}\left(\frac{\partial M}{\partial T}\right)_{B} d B .
$$

Besides the above MCE property characteristics, in practical applications the refrigeration efficiency factor $\eta$ and hold time are important quantities measuring their cooling performance. The efficiency factor is defined as the ratio $\eta=\Delta Q_{c} / \Delta Q_{m}$, where $\Delta Q_{c}=\int_{T_{f}}^{T_{i}} T(\partial S / \partial T)_{B_{f}} d T$ refers to the heat absorption from load (indicated by the red shadow area ACDE in Fig. 1), and $\Delta Q_{m}=T_{i}\left[S\left(B_{f}, T_{i}\right)-S\left(B_{i}, T_{i}\right)\right]$
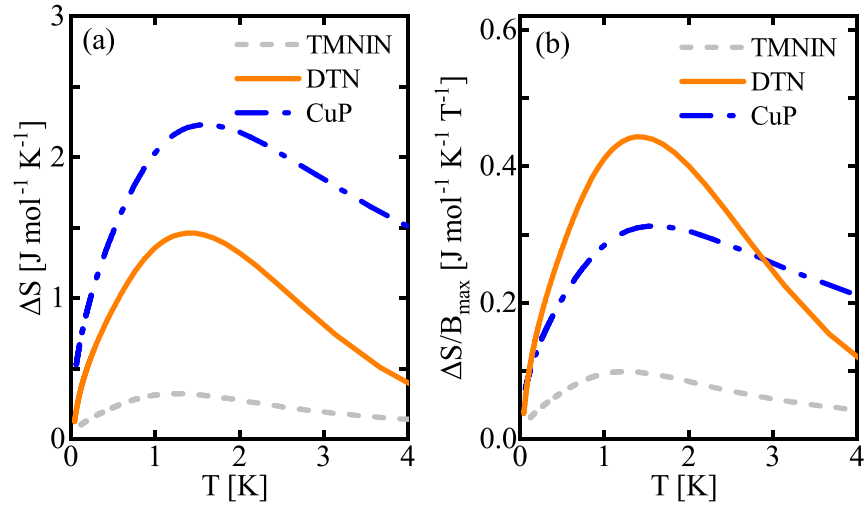

FIG. 2. (a) Simulated molar magnetic entropy change $\Delta S$ as a function of temperature $T$, for three compounds: TMNIN (gray dashed line), DTN (orange line), and CuP (blue dash-dotted line). (b) Comparison of the entropy change per Tesla, $\Delta S / B_{\max }$, for three compounds.

is the heat exchange between the material and the heat reservoir at the high temperature $T_{i}$. In multistage single-shot or continuous ADRs, the whole system must be optimized according to the precooling requirements and total weight, in which the efficiency factor is crucial [7]. In addition, the hold time-reflecting the temperature-time curve of the refrigerant in contact with the constant heat load-is another important parameter for an efficient refrigeration. The refrigerant temperature is defined as $T_{S}(t)=T_{S}(0)+\dot{Q} / C_{m}$, and particularly we require that the refrigerant temperature does not increase too rapidly (thus a long hold time) under a constant heat load $Q$.

\section{Spin-1 chain quantum magnets}

Distinct from the spin-1/2 chains, the spin-1 HAFC system has gapped ground states [27,28] with symmetry-protected topological (SPT) order [47]. There has been continuous research interest in the investigation of the spin-1 chain materials, with some prominent examples listed in Table I. In these compounds, there exists the single-ion anisotropy term $D$ [cf. Eq. (1)] besides the Heisenberg interaction $J$. In this work, we are particularly interested in the compounds TMNIN [30-33] and DTN [23,34-38], due to their very moderate

TABLE I. Some common spin-1 chain compounds and their microscopic Hamiltonian parameters, including the intrachain exchange $J$, uniaxial single-ion anisotropy $D$, the spin excitation gap $\Delta$, the Landé factor $g$, and the (lower) critical field $B_{c}$. The magnets are listed in

\begin{tabular}{|c|c|c|c|c|c|c|c|}
\hline Compound & Abbr. & $J / k_{B}(\mathrm{~K})$ & $D / k_{B}(\mathrm{~K})$ & $\Delta / k_{B}(\mathrm{~K})$ & $g$ & $B_{c}(\mathrm{~T})$ & Reference \\
\hline$\left(\mathrm{CH}_{3}\right)_{4} \mathrm{NNi}\left(\mathrm{NO}_{2}\right)_{3}$ & TMNIN & $11.7-12$ & & $4.1-4.5$ & $2.22-2.25$ & 3 & [30-33] \\
\hline $\mathrm{NiCl}_{2}-4 \mathrm{SC}\left(\mathrm{NH}_{2}\right)_{2}$ & DTN & 2.2 & 8.9 & 3.2 & 2.26 & $2.13^{\mathrm{a}}$ & {$[23,34-38]$} \\
\hline $\mathrm{Ni}\left(\mathrm{C}_{2} \mathrm{H}_{8} \mathrm{~N}_{2}\right)_{2} \mathrm{NO}_{2} \mathrm{BF}_{4}$ & NENB & $44.8-47.7$ & 7.5 & 17.4 & 2.14 & $9-13$ & {$[15,39]$} \\
\hline $\mathrm{Ni}\left(\mathrm{C}_{2} \mathrm{H}_{8} \mathrm{~N}_{2}\right)_{2} \mathrm{NO}_{2} \mathrm{ClO}_{4}$ & NENP & $46.2-48$ & $10-16$ & $13-7$ & $2.1-2.2$ & $9.8-13.4$ & {$[40,41]$} \\
\hline $\mathrm{Ni}\left(\mathrm{C}_{3} \mathrm{H}_{10} \mathrm{~N}_{2}\right)_{2} \mathrm{NO}_{2} \mathrm{ClO}_{4}$ & NINO & $50-52$ & $11.5-16$ & $10-15$ & 2.2 & $8.9-11.2$ & {$[42,43]$} \\
\hline $\mathrm{Ni}\left(\mathrm{C}_{3} \mathrm{H}_{10} \mathrm{~N}_{2}\right)_{2} \mathrm{~N}_{3} \mathrm{ClO}_{4}$ & NINAZ & $100-145$ & & $30-44$ & & & {$[31,44]$} \\
\hline $\mathrm{Y}_{2} \mathrm{BaNiO}_{5}$ & & $250-280$ & $50-56$ & $60.5-110$ & 2.16 & & {$[45,46]$} \\
\hline
\end{tabular}
ascending order in strength of the Heisenberg coupling $J$.

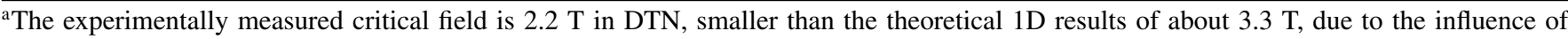
interchain interactions. 
critical field strength $B_{c} \leqslant 3 \mathrm{~T}$ (cf. Table I) that is very suitable for magnetic refrigeration applications. Besides these two members, there are other spin- 1 chain materials in the family, including NENB [15,39], NENP [40,41], and NINO [42,43] (cf. Table I), which are very common frustration-free spin-1 materials that can be described by the Hamiltonian given by Eq. (1).

Despite a little dispute about the specific value of $J$ and Landé factor $g$, consensus has been reached that the compound TMNIN can be well described by a spin-1 HAFC with negligible single-ion anisotropy [30,33], through fitting the magnetic susceptibility [30], specific heat [32], and magnetization curve [31]. Below, to explore the MCE properties of materials with the corresponding theoretical model, we take the parameter set $J=12 \mathrm{~K}$ and $g=2.25$ from Ref. [30] for TMNIN, and choose the set of parameters $J=2.2 \mathrm{~K}, D=8.9 \mathrm{~K}$, and $g=$ 2.26 for DTN [35]. Before discussing the iMCE properties and performances of the spin-1 chain refrigerants, we note that TMNIN can be regarded as an excellent spin-1 HAFC material that corresponds to a gapped ground state, while the DTN has $D / J \simeq 4$ and resides well in the trivial large$D$ phase. TMNIN opens up the spin excitation gap due to the emergence of SPT order, in sharp distinction to gapless spin-1/2 materials such as CuP. Therefore, it is interesting to compare the iMCE of these two materials as topological Haldane vs trivial large- $D$ magnetic refrigerants.

\section{INVERSE MCE IN THE SPIN-1 CHAIN MATERIALS}

Below we provide our main results of the magnetothermodynamics of two spin-1 chain magnets, i.e., the Haldane chain TMNIN and large- $D$ chain DTN, and compare them to the spin-1/2 HAFC compound $\mathrm{CuP}$ (with parameters $J=$ $3.2 \mathrm{~K}$ and $g=2.33$ [48]). We show the MCE characteristics, including the adiabatic temperature change $\Delta T_{\mathrm{ad}}$, isothermal entropy change $\Delta S$, Güneisen parameter $\Gamma_{B}$, and differential characterization $\Theta_{T}$, etc. The practical cooling performance such as the efficiency factor $\eta$ and hold time are also discussed and compared in this section.

\section{A. Entropy curves and isothermal entropy change $\Delta S$}

Magnetic fields can tune the spin states of the system and induce significant entropy change that can then be transferred into cooling effects. In Fig. 1, we show the entropy curves at two magnetic fields - the initial field $B_{i}$ and the final field $B_{f}$. As we are considering the iMCE process, they are set as $B_{i}=0, B_{f}=3.26 \mathrm{~T}$ (TMNIN) and $B_{i}=0, B_{f}=3.3 \mathrm{~T}$ (DTN) for the two spin-1 compounds. It should be noted that in the iMCE process, the largest required field (here $B_{f}$ ) is to the right of the critical field value $\left(B_{c}\right)$. This is in sharp contrast to the MCE process of spin-1/2 material $\mathrm{CuP}$, where $B_{f}=4.09 \mathrm{~T}$ (also at the QCP) and the largest field in the cooling procedure is instead $B_{i}=7.14 \mathrm{~T}$, much greater than that required in the compounds TMNIN and DTN. Such significant reduction of the maximal magnetic fields is important for the implementation of the quantum magnetic refrigeration in, say, the space applications.

From the thermal entropy curves in Fig. 1, we find that the Haldane magnet TMNIN has a rather small isothermal entropy change $\Delta S$, clearly less than $1 \mathrm{~J} \mathrm{~mol}^{-1} \mathrm{~K}^{-1}$ regardless of the working temperature (cf. Fig. 2). However, the large- $D$ magnet DTN is found to conduct a quite prominent entropy change, as seen in Figs. 1 and 2, comparable to that of the spin- $1 / 2$ compound CuP. As DTN only requires a maximal field $\left(B_{\max }=B_{f}=3.3 \mathrm{~T}\right)$, half of that for $\mathrm{CuP}$, we find DTN has the highest "efficiency," i.e., entropy change per Tesla, over the other two compounds, as shown in Fig. 2(b).

\section{B. Isentropes and adiabatic temperature change $\Delta T_{\text {ad }}$}

In the $\mathrm{iMCE}$ cooling procedure, the compounds are first magnetized along the isothermal line $\mathrm{AB}$ (red arrowed line in Fig. 1). A larger $\Delta S$ means the greater cooling capacity, which, in the adiabatic process (indicated by the horizontal line $\mathrm{BC}$ ), is translated into a large temperature change, $\Delta T_{\mathrm{ad}}$. When the magnet reaches its lowest temperature $T_{f}$ at the point $\mathrm{C}$, we contact the refrigerant with the heat load and it starts to absorb heat from there. The temperature of the magnetic refrigerant gradually rises up along the isofield line CA with a fixed field $B_{f}$.

On this basis, it becomes very meaningful to compute the isentropes of the three compounds and determine the adiabatic temperature change $\Delta T_{\text {ad }}$ from there. In Figs. 3(a) and 3(b), we show isentropes of TMNIN and DTN, where the criticality-enhanced iMCE can be clearly observed. When the magnetic field increases from zero $\left(B_{i}=0\right)$ to critical field ( $B_{f} \geqslant B_{c} \simeq 3$ T for both spin-1 compounds), we find that the temperature decreases monotonically, e.g., from about $2 \mathrm{~K}$ to about $1 \mathrm{~K}$ (for TMNIN) and $500 \mathrm{mK}$ (DTN). The $\Delta T_{\mathrm{ad}}$ results are collected and shown in Fig. 4, which are found to be significant in the course of increasing fields for both TMNIN and DTN, in a wide range of initial temperatures $T_{i}$, shown up to $2.5 \mathrm{~K}$.

In the isentropes of DTN (and also TMNIN) in Fig. 3, we can recognize two dips in low- $T$ isentropic lines, which correspond to the two field-driven QCP. The lower-field one (at about 3.3 T for DTN) has strong iMCE occurring due to the closure of the spin gap, while the one at the higher field (about $11.7 \mathrm{~T}$ for DTN) is the saturation transition where the spins become polarized. Different from the spin- 1 chains, the results of spin-1/2 CuP in Fig. 3(c) show only one saturation QCP at a field of about $B_{c} \simeq 4 \mathrm{~T}$. If we increase the fields from zero to $B_{c}$, the iMCE in CuP is apparently weak, while the MCE in CuP is significant when decreasing from a large $B_{i}$ higher than $7 \mathrm{~T}$ [cf. Figs. 3(c) and 4(a)]. Therefore, to make a fair comparison, we compute the temperature change $\Delta T_{\text {ad }}$ per Tesla, i.e., $\Delta T_{\text {ad }} / B_{\max }$, in Fig. 4(b). From the same (high) initial temperatures $T_{i} \lesssim 2.5 \mathrm{~K}$, we find that the DTN actually has the largest ratio, and TMNIN is also more efficient than CuP.

\section{Güneisen parameter $\Gamma_{B}$ and differential iMCE $\Theta_{T}$}

Above we have computed the isothermal entropy change $\Delta S$ and adiabatic temperature change $\Delta T_{\text {ad }}$ of two spin-1 materials and find that the absolute and per-Tesla values are both important for characterizing iMCE properties. As a matter of fact, to compare the MCE properties more faithfully by getting rid of the influences of different field ranges, we 

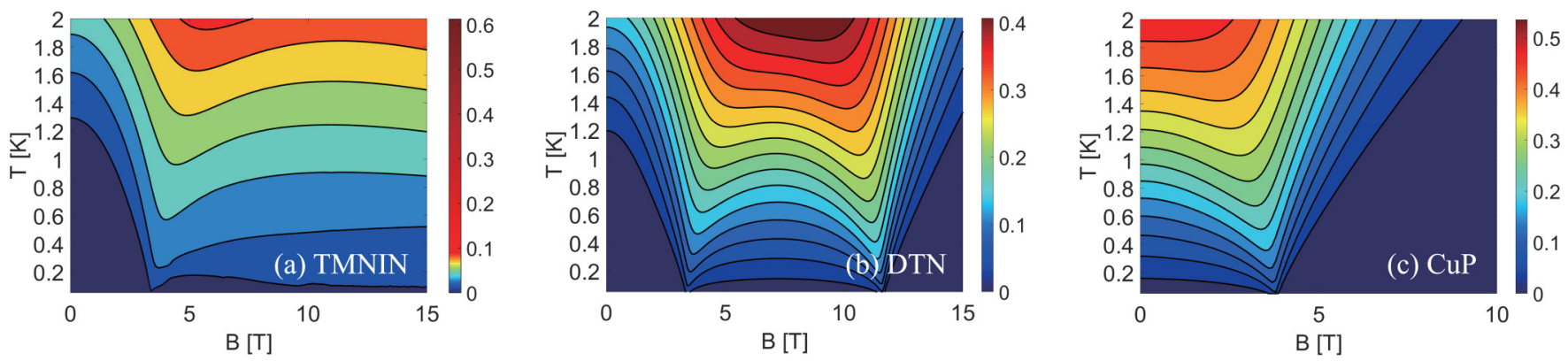

FIG. 3. Simulated isentropic contour plots of three spin-chain compounds, including the spin-1 materials (a) TMNIN and (b) DTN, and (c) the spin-1/2 chain CuP. There are two QCPs at lower $\left(B_{c}\right)$ and upper $\left(B_{s}\right)$ critical fields in (a) and (b), where $B_{c}=3.26 \mathrm{~T}$ (and $B_{s} \simeq 32 \mathrm{~T}$, not shown) [49] for TMNIN and $B_{c}=3.3 \mathrm{~T}, B_{s}=11.7 \mathrm{~T}$ for DTN. Between $B_{c}$ and $B_{s}$, there exists a continuous Tomonaga-Luttinger liquid (TLL) regime with relatively flat isentropic lines at low temperature.

exploit the differential characterizations including the Grüneisen parameter $\Gamma_{B}$ and differential entropy change $\Theta_{T}$. With these, we are able to compare the MCE properties point by point at each magnetic field.

The Grüneisen ratios $\Gamma_{B}$ of three spin-chain materials are compared in Fig. 5. In all three cases, we find pronounced peaks in $\Gamma_{B}$ that change its sign abruptly near the QCP $[11,12]$, revealing the quantum criticality-enhanced MCE. The height of the $\Gamma$ peak represents the adiabatic temperature change rate under an infinitesimal field change, which increases as $T$ lowers and diverges as $T \rightarrow 0$ [11]. Again, we see that the $\Gamma_{B}$ peaks of TMNIN are much weaker than those of DTN and CuP at the same temperature. The positive peaks and negative dips represent, respectively, the MCE and iMCE in the materials, which are quite different for the spin-1 DTN and spin- $1 / 2 \mathrm{CuP}$ materials. For DTN, $\Gamma_{B}$ is negative (iMCE) in the small-field side and positive (MCE) on the other, with the negative dip much more pronounced as compared to the positive peak, i.e., the iMCE in DTN is much stronger than MCE. On the contrary, CuP exhibits exactly the reverse behaviors, i.e., with a pronounced MCE peak to the right of the critical field, while a very weak iMCE peak on the left. This can be understood as the TLL phase appears at different sides of the QCP in the two compounds CuP and DTN.
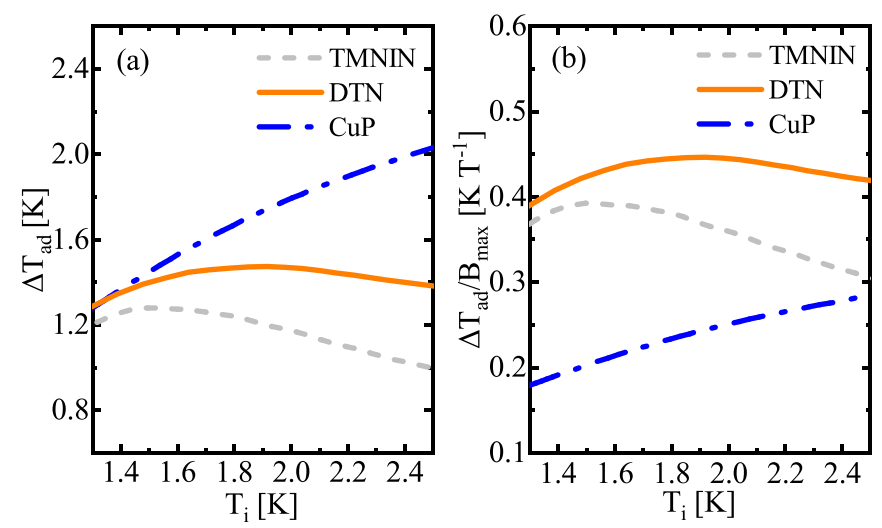

FIG. 4. (a) The adiabatic temperature change $\Delta T_{\text {ad }}$ and (b) temperature change per Tesla, $\Delta T_{\mathrm{ad}} / B_{\max }$, of the three spin-chain compounds considered in this work.
The results of the entropy change rate $\Theta_{T}$ are shown in Fig. 6, which presents the clear positive peaks (iMCE) and negative dip (MCE) around the critical fields at low temperature. Similar to the observations in $\Gamma_{B}$, TMNIN again have only rather weak peaks (dips) in $\Theta_{T}$, while DTN has a pronounced peak with a height of a similar magnitude to the dip in the CuP curve [Fig. 6(c)], showing strong iMCE. Both $\Gamma_{B}$ and $\Theta_{T}$ display characteristic divergent behaviors close to the QCP and change signs as the field crosses the critical point, indicating the pronounced refrigeration effects through adiabatic demagnetization (MCE) and magnetization (iMCE).

\section{D. iMCE performance: Efficiency factor $\boldsymbol{\eta}$ and hold time}

In practical applications, the refrigeration efficiency factor $\eta$ and the hold time are of significance to maintain a sustainable and high-efficiency refrigeration procedure. The efficiency factor $\eta$ is the ratio between heat absorbed $Q_{c}$ from the heat load (i.e., the area of the dashed line between the $\mathrm{AC}$ and DE lines in Fig. 1) and the released heat $Q_{m}$ to the heat reservoir, i.e., $\eta=Q_{c} / Q_{m}$. For DTN, the area of the red shadow in Fig. 1 is $\Delta Q_{c}=0.915 \mathrm{~J} / \mathrm{mol}$, similar to that of the spin- $1 / 2$ material CuP with $\Delta Q_{c}=1.144 \mathrm{~J} / \mathrm{mol}$, while requiring less than half the field. For the Haldane refrigerant TMNIN, we see a small heat absorption $\Delta Q_{c}=0.227 \mathrm{~J} / \mathrm{mol}$, again outperformed by the large- $D$ magnet DTN. Besides the heat absorption, we are also interested in the heat release $Q_{m}$ in the isothermal process (area of the rectangle ABDE), as there heat has to be expelled to the outer environment and thus constitutes a load for, e.g., mechanical cooling or higher-stage ADR. For a robust and efficient refrigeration system, we want the refrigerant to absorb $Q_{c}$ as large as possible while, at the same time, releasing a smaller amount of heat to the environment, i.e., to have a high efficiency factor $\eta[7,8]$. From Fig. 1, we find that such a rate of the compound DTN is $\eta=47.5 \%$ when working between $T_{i}=1.3$ and $T_{f}=0.09 \mathrm{~K}$, which is considerably higher than that of $\mathrm{CuP}(26 \%$ from the same initial temperature $T_{i}$ as reported in Refs. [16,17]).

After the adiabatic demagnetization process (BC line in Fig. 1), the refrigerant temperature reaches the lowest value $T_{f}$ at the magnetic field $B_{f}=B_{c}$. After that, the refrigerant contacts with the load and its temperature rises along the isofield line CA. We want the refrigerant with good performance to absorb heat without warming up too rapidly. The simulated 

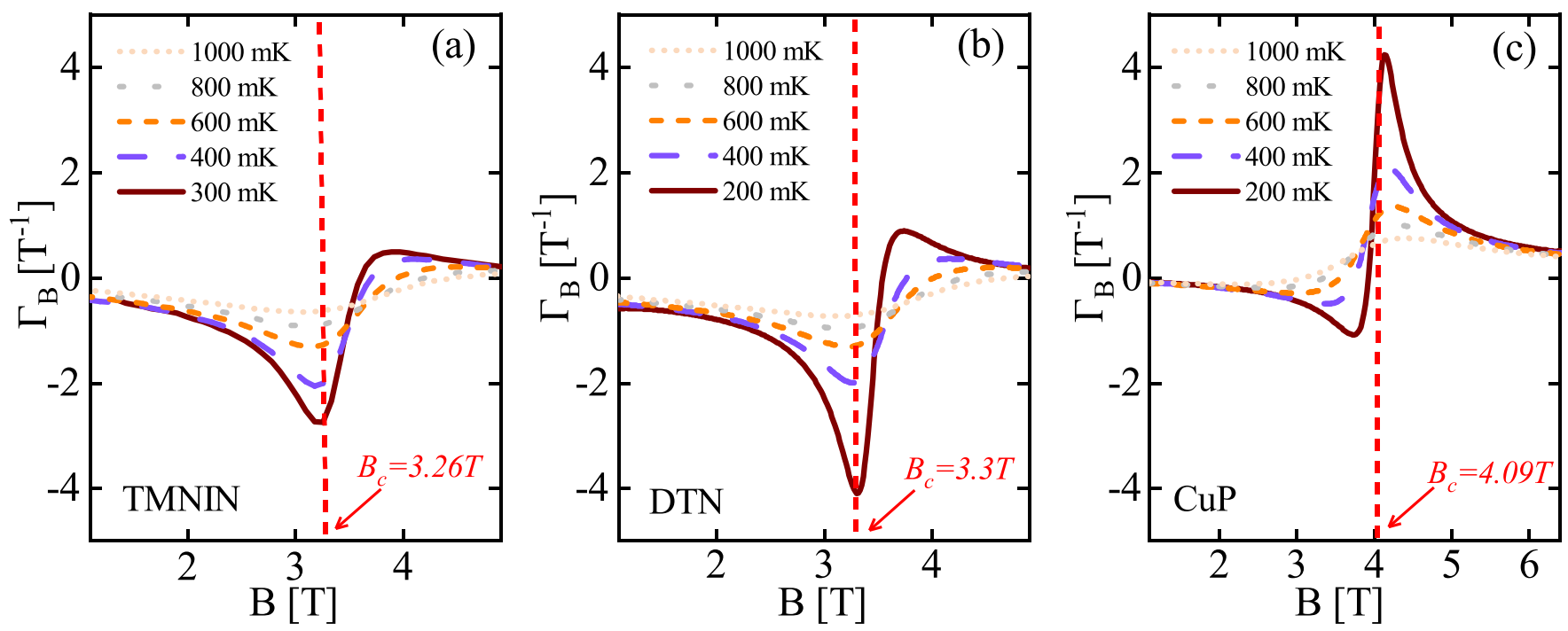

FIG. 5. The simulated Grüneisen parameter $\Gamma_{B}$ of three materials: (a) DTN, (b) CuP, and (c) TMNIN, shown at various temperatures from $1000 \mathrm{mK}$ down to $200 \mathrm{mK}$. The red vertical dashed line indicates the critical magnetic fields of the three spin-chain materials.

temperature $T_{S}$ at time $t$ is

$$
T_{S}(t)=T_{S}(0)+\dot{Q} / C_{m},
$$

which mainly depends on the magnetic specific heat $C_{m}$ of the refrigerant and heat load $\dot{Q}$. We assume that the heat is transferred at a constant rate $\dot{Q}=5 \mu \mathrm{W}$ typical for space applications [16], and start from an initial temperature $T_{S}(0)=$ $0.01 J / k_{\mathrm{B}}$, where $J$ is the spin coupling constants of the compound. The results, i.e., temperature $T_{S}(t)-T_{S}(0)$ versus time $t$, of the three compounds are shown in Fig. 7, from which we find that the spin-1 DTN has a very similar hold time as that of the spin-1/2 CuP. The hold time of the latter has been shown to be quite competitive as compared to commonly used paramagnetic salts [16]. Overall, we find that the spin-1 magnetic refrigerant DTN provides excellent MCE performance in terms of efficiency factor $\eta$ and hold time.

\section{DISCUSSION AND OUTLOOK}

Clearly distinct from the paramagnetic ADR where only isolated ions are involved in the demagnetization cooling process, here in quantum magnetic refrigeration we exploit the correlation and entanglement of the spins and the strong quantum fluctuations as our resource of cooling capacity, and significant temperature decrease can be gained in both demagnetization (MCE) and magnetization (iMCE) processes. Moreover, different from the conventional (classical) MCE that is most prominent near thermal Curie phase transition at finite temperature, quantum refrigeration is strongly enhanced near the quantum phase transitions at $T=0$. The strongly fluctuating thermal states near the quantum critical point prevent the constituents, i.e., spins, from freezing at low temperatures and even until zero temperatures, resulting in
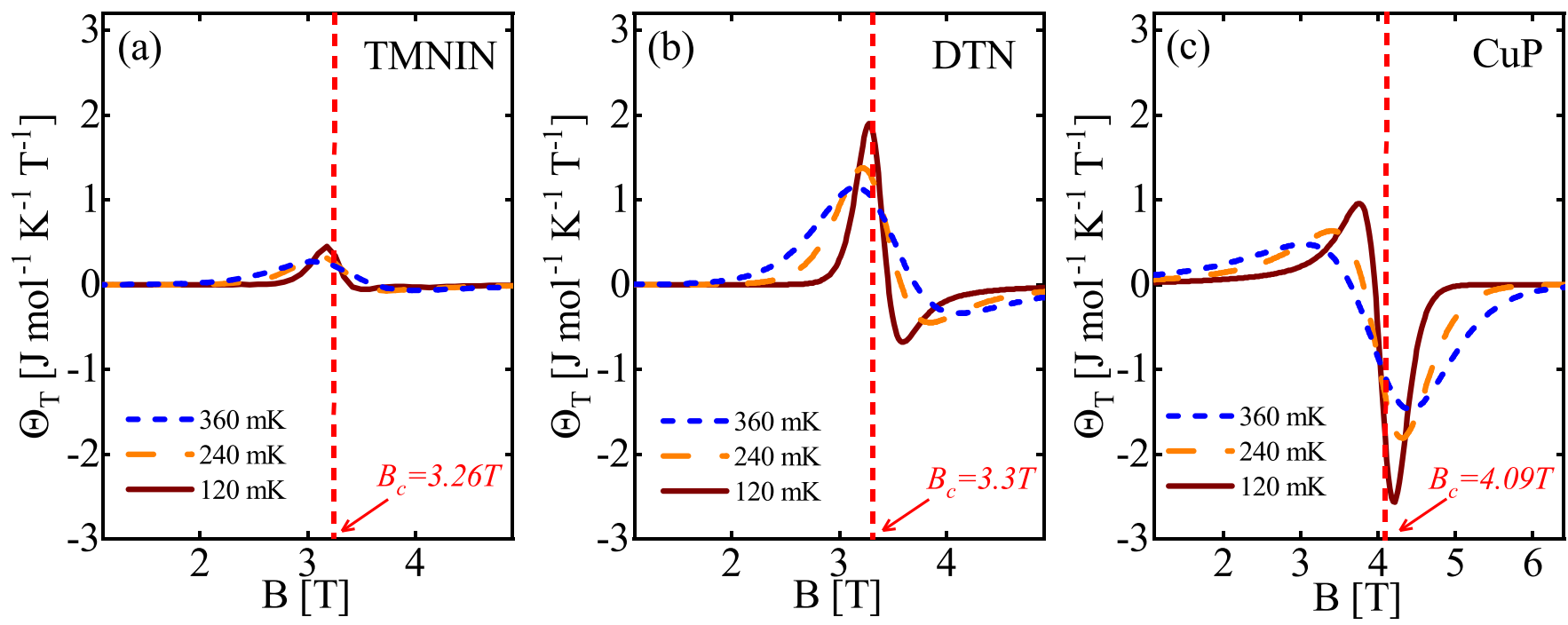

FIG. 6. The simulated entropy change rate $\Theta_{T}$ of three materials: (a) DTN, (b) CuP, and (c) TMNIN, shown at various temperatures, from 360 down to $120 \mathrm{mK}$. 


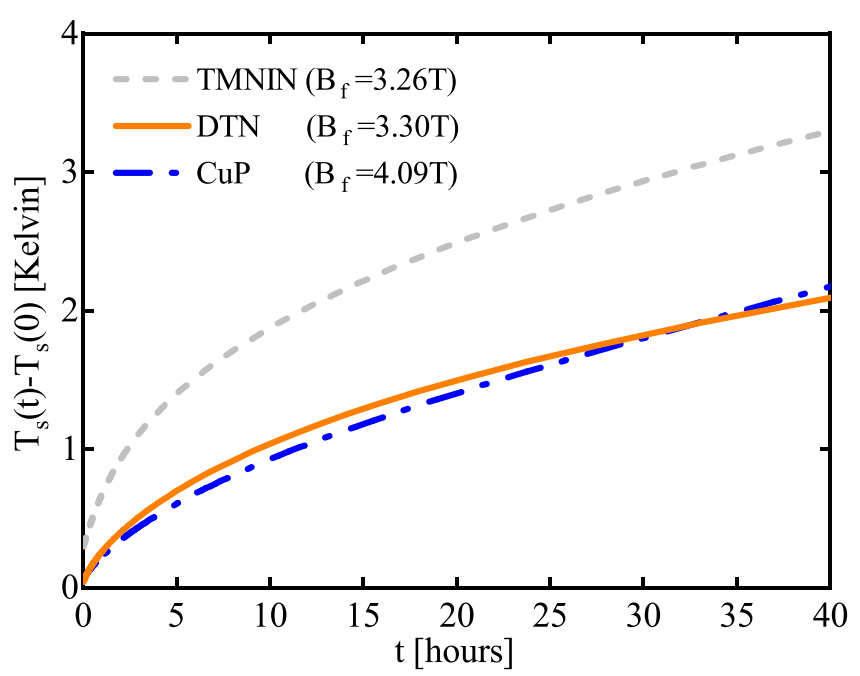

FIG. 7. The sample temperature $T_{s}$ as a function of time $t$ at a fixed field $B=B_{f}$, calculated under a constant heat load of $5 \mu \mathrm{W}$. We consider a $100 \mathrm{~g}$ substance of the spin-1/2 CuP (blue dashed line), TMNIN (gray dashed line), and DTN (orange solid line) in the calculations.

large entropy change and divergent differential refrigeration characteristics $\Gamma_{B}$ and $\Theta_{T}$, etc., as $T$ goes to zero.

Here we have systematically investigated the iMCE near the field-induced quantum critical point in the spin-1 quantum magnets. As these compounds have a finite spin gap either due to the Haldane topological origin (in TMNIN) or large single-ion effects (DTN), significant iMCEs have been observed when the spin gap is closed. In particular, the latter is found to have comparable cooling capacities and even better performance as compared to the criticality-enhanced $\mathrm{MCE}$ material $\mathrm{CuP}$, with considerably reduced magnetic fields required.

Moreover, in the compound DTN, the field-induced quantum phase transition can be described as a Bose-Einstein condensation in quantum magnets, and has a high thermal conductivity even at very low temperature [23]. As the typical paramagnetic salts have low thermal conductivity since the spins do not talk to each other in the "gas" states, here in the spin-1 compounds heat can be transferred through the magnetic excitations between the coupled spins. This renders the spin-1 magnet DTN as a very promising quantum magnetic refrigerant with both high cooling capacities and excellent performance.

The compounds DTN and TMNIN are very classic spin-1 chain materials and their single-crystal samples have been synthesized for quite some time. For example, a high-quality single crystal of DTN has been used in the studies of Bose-Einstein condensation in quantum magnets $[34,35]$ and thermal transport measurements $[23,37]$. Therefore, above we only consider the iMCE in single-crystal DTN samples, and the fields are applied along the single-ion $c$ axis. In the case that only powder samples are available or when the field tilts at an angle with respect to the single-crystal $c$ axis, we need to consider the iMCE properties of DTN under a tilted field. We have also performed the calculations and found that the

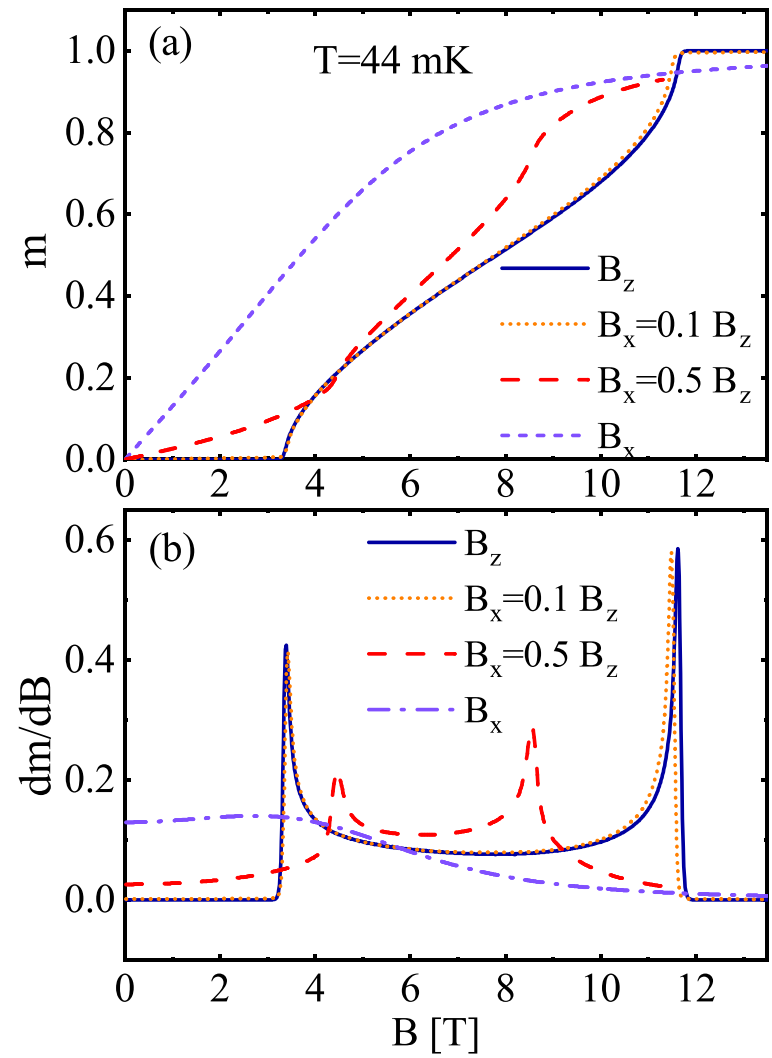

FIG. 8. (a) Low-temperature magnetization curves under magnetic fields along various directions, including the $c$ axis (longitudinal field $B_{z}$ ), tilted directions $B_{x} / B_{z}=0.1,0.5$, and the transverse field $\left(B_{x}\right)$. (b) The derivative $d m / d B$ of the three cases.

field-induced QCP as well as enhanced iMCE are still present for a range of tilting angles (see Appendix B).

Lastly, normal and inverse MCE properties for magnets with high Curie temperatures have been intensively discussed for room- or near-room-temperature refrigeration [50-54], which helps one to enhance the cooling capacity and design compact continuous refrigeration machinery [55]. Similarly, the efficient iMCE refrigerant, e.g., spin-1 DTN here, is important for designing a low- $T$ continuous cooling cycle where the temperature can be decreased in both the magnetization and demagnetization processes. Our work fills this gap by finding DTN to be a very promising iMCE compound that provides a high-performance refrigerants in the "arsenal" of spin-chain quantum materials.

\section{ACKNOWLEDGMENTS}

This work was supported by the National Natural Science Foundation of China (Grants No. 11704113, No. 11834014, No. 11974036, No. 12074024), Natural Science Foundation of Hunan Province, China (Grant No. 2018JJ3111), and the Scientific Research Fund of Hunan Provincial Education Department of China (Grant No. 19B159).

\section{APPENDIX A: LINEARIZED TENSOR RENORMALIZATION GROUP METHOD}

The thermodynamics of the spin-chain models and materials can be calculated via the thermal-state tensor 

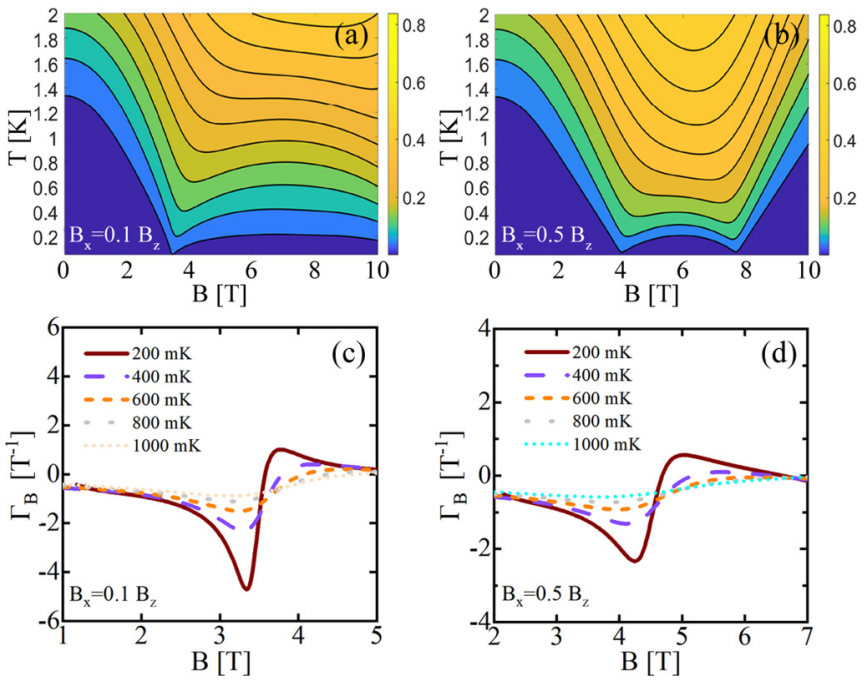

FIG. 9. (a),(b) The simulated isentropic contours and (c),(d) the Grüneisen parameters under tilted fields $\left(B_{x} / B_{z}=0.1\right.$ and 0.5$)$. The lower critical fields are slightly modified in the two cases, and the $\Gamma_{B}$ dip characterizing iMCE becomes less pronounced for $B_{x}=0.5 B_{z}$ as compared to that of $B_{x}=0.1 B_{z}$.

renormalization group (TRG) methods. In this work, we employ the linearized TRG (LTRG) $[21,22]$ proposed by some of the authors to perform the finite- $T$ simulations. For the spin-1 chain model given by Eq. (1), the Hamiltonian can be divided into odd and even parts through the Trotter-Suzuki decomposition [56], and the thermal density matrix can be expressed as

$$
\hat{\rho}_{\beta}=e^{-\beta H}=\left(e^{-\tau H}\right)^{n}=\simeq\left(e^{-\tau H_{\text {odd }}} e^{-\tau H_{\text {even }}}\right)^{n},
$$

where $n$ is a sufficiently large integer and the (small) imaginary time slice is $\tau=\beta / n$. In practice, $\tau$ is chosen as 0.05 and we iteratively project the imaginary evolution gates $e^{-\tau H}$ of a single Trotter step to the matrix-product density operator, so as to cool down the temperature. In the bilayer algorithm, the density matrix at an inverse temperature $\beta$ is obtained by

$$
\hat{\rho}_{\beta}=\hat{\rho}_{\beta / 2}^{\dagger} \cdot \hat{\rho}_{\beta / 2},
$$

which saves the cost of calculations (by half) and considerably improves the accuracy by assuring positivity of the density matrix [22].

\section{APPENDIX B: QUANTUM PHASE TRANSITION AND MAGNETOCALORIC EFFECTS UNDER TILTED FIELDS}

When only powder samples are available or a small misalignment of the field direction occurs for a single-crystal sample, the iMCE properties of DTN need to be reconsidered as the fields can be applied along directions other than the single-ion $c$ axis. In Fig. 8, we show the low- $T$ magnetization process along the tilted directions as described by the Hamiltonian

$$
\mathcal{H}=\sum_{i} J \vec{S}_{i} \cdot \vec{S}_{i+1}+D\left(S_{i}^{z}\right)^{2}+g \mu_{B}\left(B_{x} S_{i}^{x}+B_{z} S_{i}^{z}\right),
$$

with the total field strength $B=\sqrt{B_{x}^{2}+B_{z}^{2}}$. In Fig. 8, we computed the cases $B_{x}=0.1 B_{z}\left(\theta=5.71^{\circ}\right)$ and $B_{x}=0.5 B_{z}$ $\left(\theta=26.6^{\circ}\right)$, and find there still exists criticality-enhanced iMCE when tilting the longitudinal field from the $c$ axis with an angle $\theta$. Correspondingly, in Fig. 9 we clearly see the iMCE near the field-induced QCPs, including the evident temperature decrease and clear dip in the Grüneisen parameter $\Gamma_{B}$.

On the other hand, when the field is applied perpendicular to the single-ion axis (i.e., $B_{x}$ with $\theta=90^{\circ}$ ), indeed we observe that the QCP is absent (Fig. 8) and thus no pronounced iMCE can be observed. Based upon these calculations, we find that the quantum criticality enhanced iMCE exists for certain tilted fields, but not for too large tilting angles (e.g., a perpendicular $B_{x}$ ). Nevertheless, when the tilting angle is small (e.g., $\theta=5.71^{\circ}$ ), we find the iMCE rather robust and barely changed as compared to that along the $c$ axis.
[1] E. Warburg, Magnetic investigations, Ann. Phys. Berlin 249, 141 (1881).

[2] P. Weiss and A. Piccard, Magnetocaloric phenomenon, J. Phys. (Paris) 7, 103 (1917).

[3] O. Tegus, E. Brück, K. H. J. Buschow, and F. R. de Boer, Transition-metal-based magnetic refrigerants for roomtemperature applications, Nature (London) 415, 150 (2002).

[4] A. Smith, Who discovered the magnetocaloric effect?, Eur. Phys. J. H 38, 507 (2013).

[5] O. V. Lounnasmaa, Experimental Principles and Methods Below $1 K$ (Academic, London, 1974).

[6] C. Hagmann and P. L. Richards, Adiabatic demagnetization refrigerators for small laboratory experiments and space astronomy, Cryogenics 35, 303 (1995).

[7] P. J. Shirron, Cooling capabilities of adiabatic demagnetization refrigerators, J. Low Temp. Phys. 148, 915 (2007).

[8] A. E. Jahromi, P. J. Shirron, and M. J. DiPirro, Sub-Kelvin Cooling Systems for Quantum Computers, Tech. Rep. no. GSFC-E-DAA-TN70637 (NASA Goddard Space Flight Center, Greenbelt, MD, 2019).
[9] M. E. Zhitomirsky, Enhanced magnetocaloric effect in frustrated magnets, Phys. Rev. B 67, 104421 (2003).

[10] M. E. Zhitomirsky and A. Honecker, Magnetocaloric effect in one-dimensional antiferromagnets, J. Stat. Mech.: Theory Expt. (2004) P07012.

[11] L. J. Zhu, M. Garst, A. Rosch, and Q. M. Si, Universally Diverging Grüneisen Parameter and the Magnetocaloric Effect Close to Quantum Critical Points, Phys. Rev. Lett. 91, 066404 (2003).

[12] M. Garst and A. Rosch, Sign change of the Grüneisen parameter and magnetocaloric effect near quantum critical points, Phys. Rev. B 72, 205129 (2005).

[13] A. Honecker and S. Wessel, Magnetocaloric effect in quantum spin-s chains, Condens. Matter Phys. 12, 399 (2009).

[14] J. W. Sharples, D. Collison, E. J. L. McInnes, J. Schnack, E. Palacios, and M. Evangelisti, Quantum signatures of a molecular nanomagnet in direct magnetocaloric measurements, Nat. Commun. 5, 5321 (2014).

[15] M. Orendáč, R. Tarasenko, V. Tkáč, A. Orendáčová, and V. Sechovský, Specific heat study of the magnetocaloric 
effect in the Haldane-gap $\mathrm{S}=1$ spin-chain material $\left[\mathrm{Ni}\left(\mathrm{C}_{2} \mathrm{H}_{8} \mathrm{~N}_{2}\right)_{2} \mathrm{NO}_{2}\right]\left(\mathrm{BF}_{4}\right)$, Phys. Rev. B 96, 094425 (2017).

[16] B. Wolf, Y. Tsui, D. Jaiswal-Nagar, U. Tutsch, A. Honecker, K. Remović-Langer, G. Hofmann, A. Prokofiev, W. Assmus, G. Donath, and M. Lang, Magnetocaloric effect and magnetic cooling near a field-induced quantum-critical point, Proc. Natl. Acad. Sci. 108, 6862 (2011).

[17] M. Lang, B. Wolf, A. Honecker, L. Balents, U. Tutsch, P. T. Cong, G. Hofmann, N. Krüger, F. Ritter, W. Assmus, and A. Prokofiev, Field-induced quantum criticality - Application to magnetic cooling, Phys. Status Solidi B 250, 457 (2013)

[18] U. Schollwöck, J. Richter, D. J. J. Farnell, and R. F. Bishop, Quantum Magnetism, Vol. 645 (Springer, Berlin, Heidelberg, 2004).

[19] S. Sachdev, Quantum Phase Transitions, Vol. 12 (Cambridge University Press, Cambridge, 2011).

[20] P. Gegenwart, Grüneisen parameter studies on heavy fermion quantum criticality, Rep. Prog. Phys. 79, 114502 (2016).

[21] W. Li, S. J. Ran, S. S. Gong, Y. Zhao, B. Xi, F. Ye, and G. Su, Linearized Tensor Renormalization Group Algorithm for the Calculation of Thermodynamic Properties of Quantum Lattice Models, Phys. Rev. Lett. 106, 127202 (2011).

[22] Y. L. Dong, L. Chen, Y. J. Liu, and W. Li, Bilayer linearized tensor renormalization group approach for thermal tensor networks, Phys. Rev. B 95, 144428 (2017).

[23] X. F. Sun, W. Tao, X. M. Wang, and C. Fan, Low-Temperature Heat Transport in the Low-Dimensional Quantum Magnet $\mathrm{NiCl}_{2}-4 \mathrm{SC}\left(\mathrm{NH}_{2}\right)_{2}$, Phys. Rev. Lett. 102, 167202 (2009).

[24] J. C. Bonner, Generalized Heisenberg quantum spin chains (invited), J. Appl. Phys. 61, 3941 (1987).

[25] O. Golinelli, T. Jolicoeur, and R. Lacaze, Dispersion of magnetic excitations in a spin-1 chain with easy-plane anisotropy, Phys. Rev. B 46, 10854 (1992).

[26] F. D. M. Haldane, Nonlinear Field Theory of Large-Spin Heisenberg Antiferromagnets: Semiclassically Quantized Solitons of the One-Dimensional Easy-Axis Néel State, Phys. Rev. Lett. 50, 1153 (1983).

[27] M. P. Nightingale and H. W. J. Blote, Gap of the linear spin-1 Heisenberg antiferromagnet: A Monte Carlo calculation, Phys. Rev. B 33, 659 (1986).

[28] S. R. White and D. A. Huse, Numerical renormalization-group study of low-lying eigenstates of the antiferromagnetic $S=1$ Heisenberg chain, Phys. Rev. B 48, 3844 (1993).

[29] T. Sakai and M. Takahashi, Effect of the Haldane gap on quasione-dimensional systems, Phys. Rev. B 42, 4537 (1990).

[30] V. Gadet, M. Verdaguer, V. Briois, A. Gleizes, J. P. Renard, P. Beauvillain, C. Chappert, T. Goto, K. Le Dang, and P. Veillet, Structural and magnetic properties of $\left(\mathrm{CH}_{3}\right)_{4} \mathrm{NNi}\left(\mathrm{NO}_{2}\right)_{3}$ : A Haldane-gap system, Phys. Rev. B 44, 705 (1991).

[31] T. Takeuchi, H. Hori, M. Date, T. Yosida, K. Katsumata, J. Renard, V. Gadet, and M. Verdaguer, High field magnetization of Haldane materials TMNIN and NINAZ, J. Magn. Magn. Mater. 104, 813 (1992).

[32] M. Ito, M. Mito, H. Deguchi, and K. Takeda, The numerical comparison of magnetic susceptibility and heat capacity of TMNIN with the result of a quantum Monte Carlo method for the Haldane system, J. Phys. Soc. Jpn. 63, 1123 (1994).

[33] T. Goto, T. Ishikawa, Y. Shimaoka, and Y. Fujii, Quantum spin dynamics studied by the nuclear magnetic relaxation of protons in the Haldane-gap system $\left(\mathrm{CH}_{3}\right)_{4} \mathrm{NNi}\left(\mathrm{NO}_{2}\right)_{3}$, Phys. Rev. B 73, 214406 (2006).

[34] V. S. Zapf, D. Zocco, B. R. Hansen, M. Jaime, N. Harrison, C. D. Batista, M. Kenzelmann, C. Niedermayer, A. Lacerda, and A. Paduan-Filho, Bose-Einstein Condensation of $S=1$ Nickel Spin Degrees of Freedom in $\mathrm{NiCl}_{2}-4 \mathrm{SC}\left(\mathrm{NH}_{2}\right)_{2}$, Phys. Rev. Lett. 96, 077204 (2006).

[35] A. Paduan-Filho, K. A. Al-Hassanieh, P. Sengupta, and M. Jaime, Critical Properties at the Field-Induced Bose-Einstein Condensation in $\mathrm{NiCl}_{2}-4 \mathrm{SC}\left(\mathrm{NH}_{2}\right)_{2}$, Phys. Rev. Lett. 102, 077204 (2009).

[36] O. Chiatti, S. Zherlitsyn, A. Sytcheva, J. Wosnitza, A. A. Zvyagin, V. S. Zapf, M. Jaime, and A. Paduan-Filho, Ultrasonic investigation of $\mathrm{NiCl}_{2}-4 \mathrm{SC}\left(\mathrm{NH}_{2}\right)_{2}$, J. Phys.: Conf. Ser. 150, 042016 (2009).

[37] Y. Kohama, A. V. Sologubenko, N. R. Dilley, V. S. Zapf, M. Jaime, J. A. Mydosh, A. Paduan-Filho, K. A. Al-Hassanieh, P. Sengupta, S. Gangadharaiah, A. L. Chernyshev, and C. D. Batista, Thermal Transport and Strong Mass Renormalization in $\mathrm{NiCl}_{2}-4 \mathrm{SC}\left(\mathrm{NH}_{2}\right)_{2}$, Phys. Rev. Lett. 106, 037203 (2011).

[38] C. Psaroudaki, S. A. Zvyagin, J. Krzystek, A. Paduan-Filho, X. Zotos, and N. Papanicolaou, Magnetic excitations in the spin1 anisotropic antiferromagnet $\mathrm{NiCl}_{2}-4 \mathrm{SC}\left(\mathrm{NH}_{2}\right)_{2}$, Phys. Rev. B 85, 014412 (2012).

[39] E. Čižmár, M. Ozerov, O. Ignatchik, T. P. Papageorgiou, J. Wosnitza, S. A. Zvyagin, J. Krzystek, Z. Zhou, C. P. Landee, B. R. Landry, M. M. Turnbull, and J. L. Wikaira, Magnetic properties of the Haldane-gap material $\left[\mathrm{Ni}\left(\mathrm{C}_{2} \mathrm{H}_{8} \mathrm{~N}_{2}\right)_{2} \mathrm{NO}_{2}\right]\left(\mathrm{BF}_{4}\right)$, New J. Phys. 10, 033008 (2008).

[40] J. P. Renard, M. Verdaguer, L. P. Regnault, W. A. C. Erkelens, J. Rossat-Mignod, J. Ribas, W. G. Stirling, and C. Vettier, Quantum energy gap in two quasi-one dimensional $S=1$ Heisenberg antiferromagnet, J. App. Phys. 63, 3538 (1988).

[41] T. Kobayashi, Y. Tabuchi, K. Amaya, Y. Ajiro, T. Yosida, and M. Date, Heat capacities of Haldane-gap antiferromagnet NENP in magnetic field, J. Phys. Soc. Jpn. 61, 1772 (1992).

[42] T. Takeuchi, M. Ono, H. Hori, T. Yosida, A. Yamagishi, and M. Date, Magnetization measurement of NENP and NINO in high magnetic field, J. Phys. Soc. Jpn. 61, 3255 (1992).

[43] W. Tao, L. M. Chen, X. M. Wang, C. Fan, W. P. Ke, X. G. Liu, Z. Y. Zhao, Q. J. Li, and X. F. Sun, Crystal growth and characterization of Haldane chain compound $\mathrm{Ni}\left(\mathrm{C}_{3} \mathrm{H}_{10} \mathrm{~N}_{2}\right)_{2} \mathrm{NO}_{2} \mathrm{ClO}_{4}$, J. Cryst. Growth 327, 215 (2011).

[44] A. Zheludev, S. E. Nagler, S. M. Shapiro, L. K. Chou, D. R. Talham, and M. W. Meisel, Spin dynamics in the linear-chain $S=1$ antiferromagnet $\mathrm{Ni}\left(\mathrm{C}_{3} \mathrm{H}_{10} \mathrm{~N}_{2}\right)_{2} \mathrm{~N}_{3}\left(\mathrm{ClO}_{4}\right)$, Phys. Rev. B 53, 15004 (1996).

[45] K. Kordonis, A. V. Sologubenko, T. Lorenz, S.-W. Cheong, and A. Freimuth, Spin Thermal Conductivity of the Haldane Chain Compound $\mathrm{Y}_{2} \mathrm{BaNiO}_{5}$, Phys. Rev. Lett. 97, 115901 (2006).

[46] J. J. Li, Z. W. Ouyang, Y. C. Sun, X. Y. Yue, Z. C. Xia, and G. H. Rao, Magnetic enhancement and suppression of Haldane gap in nanocrystals of spin-chain $\mathrm{Y}_{2} \mathrm{BaNiO}_{5}$, J. Low Temp. Phys. 188, 11 (2017).

[47] Z.-C. Gu and X.-G. Wen, Tensor-entanglement-filtering renormalization approach and symmetry-protected topological order, Phys. Rev. B 80, 155131 (2009).

[48] A. V. Prokofiev, W. Assmus, K. Remović-Langer, V. Pashchenko, Y. Tsui, B. Wolf, and M. Lang, Crystal growth and magnetic properties of the copper coordination polymer 
$\left.\left[\mathrm{Cu}\left(\mu \mathrm{C}_{2} \mathrm{O}_{4}\right) \text { (4aminopyridine }\right)_{2}\left(\mathrm{H}_{2} \mathrm{O}\right)\right]_{n}$, Cryst. Res. Technol. 42, 394 (2007).

[49] T. Takeuchi, H. Hori, T. Yosida, A. Yamagishi, K. Katsumata, J. P. Renard, V. Gadet, M. Verdaguer, and M. Date, Magnetization process of Haldane materials TMNIN and NINAZ, J. Phys. Soc. Jpn. 61, 3262 (1992).

[50] T. Krenke, E. Duman, M. Acet, E. F. Wassermann, X. Moya, L. Manosa, and A. Planes, Inverse magnetocaloric effect in ferromagnetic Ni-Mn-Sn alloys, Nat. Mater. 4, 450 (2005).

[51] X. Moya, L. Mañosa, A. Planes, S. Aksoy, M. Acet, E. F. Wassermann, and T. Krenke, Cooling and heating by adiabatic magnetization in the $\mathrm{Ni}_{50} \mathrm{Mn}_{34} \mathrm{In}_{16}$ magnetic shape-memory alloy, Phys. Rev. B 75, 184412 (2007).

[52] V. B. Naik and R. Mahendiran, Normal and inverse magnetocaloric effects in ferromagnetic $\mathrm{Sm}_{0.6-x} \mathrm{La}_{x} \mathrm{Sr}_{0.4} \mathrm{MnO}_{3}$, J. Appl. Phys. 110, 053915 (2011).
[53] D. V. Maheswar Repaka, M. Aparnadevi, P. Kumar, T. S. Tripathi, and R. Mahendiran, Normal and inverse magnetocaloric effects in ferromagnetic $\operatorname{Pr}_{0.58} \mathrm{Sr}_{0.42} \mathrm{MnO}_{3}$, J. Appl. Phys. 113, 17A906 (2013).

[54] R. Das, P. Yanda, A. Sundaresan, and D. D. Sarma, Ground-state ferrimagnetism and magneto-caloric effects in $\mathrm{Nd}_{2} \mathrm{NiMnO}_{6}$, Mater. Res. Express 6, 116122 (2019).

[55] X. Zhang, B. Zhang, S. Yu, Z. Liu, W. Xu, G. Liu, J. Chen, Z. Cao, and G. Wu, Combined giant inverse and normal magnetocaloric effect for room-temperature magnetic cooling, Phys. Rev. B 76, 132403 (2007).

[56] M. Suzuki, Relationship between d-dimensional quantal spin systems and $(\mathrm{d}+1)$-dimensional Ising systems-equivalence, critical exponents and systematic approximants of the partition function and spin correlations, Prog. Theor. Phys. 56, 1454 (1976). 